\title{
Adopting Knowledge Management in an E-Learning System: Insights and Views of KM and EL Research Scholars
}

\author{
Md. Shiful Islam* \\ School of Knowledge Science \\ Japan Advanced Institute of Science and Technology (JAIST) \\ 1-1 Asahidai, Nomi City, Ishikawa 923-1292, Japan \\ Department of Information Science and Library Management \\ University of Dhaka \\ Dhaka-1000, Bangladesh \\ E-mail: islam@jaist.ac.jp or shifuldu@gmail.com
}

\section{Susumu Kunifuji}

Professor, School of Knowledge Science

Vice-President, Japan Advanced Institute of Science and Technology (JAIST)

1-1 Asahidai, Nomi City, Ishikawa 923-1292, Japan

E-mail: kuni@jaist.ac.jp

\section{Motoki Miura}

Department of Basic Sciences

Kyushu Institute of Technology, Japan

E-mail: miuramo@mns.kyutec.ac.jp

\section{Tessai Hayama}

School of Knowledge Science

Japan Advanced Institute of Science and Technology (JAIST)

1-1 Asahidai, Nomi City, Ishikawa 923-1292, Japan

E-mail: t-hayama@jaist.ac.jp

*Corresponding author

\begin{abstract}
The main goal of this study is to explore the insights and views from knowledge management (KM) and E-learning (EL) research scholars through a face-to- face interview for determining the relationship between KM and EL as well as for adopting KM in an EL system. We conducted this study based on the review of existing concepts and frameworks of KM and EL available in the secondary sources of information, and the findings of the face-to-face interviews of 17 (Seventeen) KM and EL research scholars from all over the world. We selected the respondents from the participants of two international conferences held in Japan and Thailand in 2010. The interviews were conducted during the break of the conferences using a carefully structured, short and open ended questionnaire. The levels of understanding of KM and EL among the research scholars were varied. The results indicate that there are some common goals and significant overlaps between the two knowledge
\end{abstract}


domains. They also delineate the significant implications and advantages of adopting KM and EL. The proposed model of this study theoretically contributes to the advancement of academic debate in both KM and EL domains. Since we interviewed only $17 \mathrm{KM}$ and EL research scholars worldwide from two international conferences held in 2010, the results of this study may not reflect the general scenario of the world. However, the study suggests that EL professionals should adopt KM and apply the techniques of $\mathrm{KM}$ to an EL system for the overall enhancement of the e-learning process. The study explored the original views and insights of the KM and EL research scholars regarding the relationship between KM and EL as well as adopting $\mathrm{KM}$ in an EL system.

Keywords: e-Learning; e-Learning System; Knowledge Management; Knowledge Management Process; Adoption Model

Biographical notes: Md. Shiful Islam is an Assistant Professor in the Department of Information Science and Library management, University of Dhaka, Bangladesh and currently a Ph.D. Candidate at School of Knowledge Science, Japan Advanced Institute of Science and Technology (JAIST), Ishikawa, Japan. He earned both BA and MA in Library and Information Science from the University of Dhaka, Bangladesh. He also obtained Master in Computer Applications (MCA) from the University of Comilla (Dhaka campus), Bangladesh. His areas of teaching and research interests include LIS education, E-learning, Knowledge management, digital library, new technologies and current trends in information systems, etc.

Dr. Susumu Kunifuji was born in 1947. He received B.E., M.E., and D.E. degrees from Tokyo Institute of Technology, in 1971, 1974, and 1994 respectively. He worked as a researcher at International Institute for Advanced Study of Social Information Science, FUJITSU Ltd. (1974-1982), was chief researcher at Institute for New Generation Computer Technology (1982-1986), Manager at International Institute for Advanced Study of Social Science, FUJITSU Ltd. (1986-1992), and Professor at School of Information Science at JAIST (1992-1998). Currently he is a Professor at school of Knowledge Science and Vice-President of JAIST. He is also a President of Japan Creativity Society and a member of JSAI, IPSJ, SICE, JCS, etc.

Dr. Motoki Miura was born in 1974. He received his B.E., M.E. and D.E. degrees in electronic engineering from University of Tsukuba, in 1997, 1999 and 2001 respectively. From August 2001 to March 2004, he worked as a research associate at TARA Center, University of Tsukuba. He worked as a research associate and Assistant Professor at JAIST from April 2004 to March 2008. Currently he is an Associate Professor at Kyushu Institute of technology, Japan. He is a member of JSAI, IPSJ, JSSST, ACM, JSET, and HIS.

Dr. Tessai Hayama received his B.E. degree in knowledge engineering from Doshisha University in 2001, and M.S. and Ph.D. degree in Knowledge Science from JAIST in 2003 and 2006 respectively. Currently he is an Assistant Professor in School of Knowledge Science at JAIST. His research Interests include creative support systems and human interface. He is a member of JSAI, IPSJ, and JSCE. 


\section{Background and Objectives}

The rapidly growing use of information and communication technology (ICT) in academia is changing the way in which knowledge is created, organized, stored, managed, and disseminated. The convergence of EL and KM fosters a constructive, open, dynamic, interconnected, distributed, adaptive, user friendly, socially concerned and accessible wealth of knowledge (Lytras, Naeve, \& Pouloudi, 2005). EL has become a significant step in the development of KM systems. Morales (2005) contended that KM and EL are closely related because EL users need a suitable KM that can help them to obtain the kind of content they need, together with as correct and complete information as possible. KM oriented EL has become the effective tool that transfers tacit knowledge information into explicit knowledge, as a result, organizations with this system can accomplish knowledge and information delivery in or between organizations (Liu \& Wang, 2009). Therefore, it becomes essential to determine the relationship between KM and EL as well as to adopt $\mathrm{KM}$ in an EL system. We made an attempt to determine the relationship between KM and EL based on the insights and views of KM and EL research scholars through a face-toface interview, and the existing literature. We also propose a KM and EL adoption model through which we could expect the conversion of tacit knowledge, the promotion of knowledge organization and retrieval, the enhancement of knowledge sharing, and the proper management of knowledge resources as the implications and benefits.

This paper first introduces the conceptual framework of the KM process and EL systems from the existing literature. Moreover, we analyze the data acquired from the interview for an in-depth understanding of KM and EL, and we explore the common goals and significant overlaps of the KM and EL domains to show the relationship between the KM and EL systems. Subsequently, we present a theoretical model for adopting KM and EL and consider the implications and expected benefits through the adoption of the KM process and EL systems. The main goal of the study is to obtain the insights and views from KM and EL research scholars through a face-to-face interview for determining the relationship between $\mathrm{KM}$ and EL as well as for adopting KM in an EL system. More specifically, the study was carried out to achieve the following objectives:

(1) Find out the common goals of KM and EL.

(2) Identify the overlaps between the two particular knowledge domains.

(3) Develop a theoretical model to adopt KM in an EL system

(4) Determine the important implications and benefits achieving from the adoption of $\mathrm{KM}$ and EL.

\section{Literature Review}

\subsection{Theoretical Framework of the KM Process}

KM can be seen as a continuous process divided into five main stages:(1) knowledge creation and acquisition (2) knowledge organization and storage, (3) knowledge distribution and integration (4) knowledge adaptation and application, and (5) knowledge evaluation, validation and refinement (see figure 1) (Vasilyeva, Pechenizkiy, \& Puuronen, 2005). According to figure 1, the given adaptation principle can be created by teachers and knowledge engineers. This adaptation principle can be suggested also as a result of 
experience in knowledge structuring and different types of presentation for the different group of students.

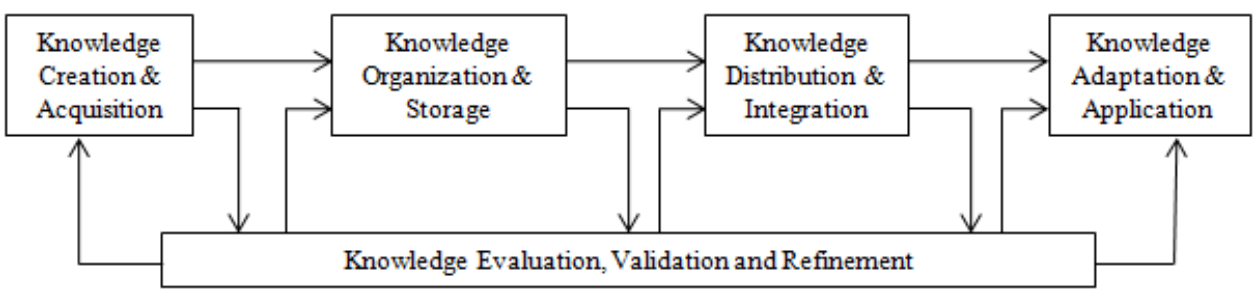

Figure 1. The Knowledge Management Process

Source: Vasilyeva, Pechenizkiy and Puuronen, (2005), p.117

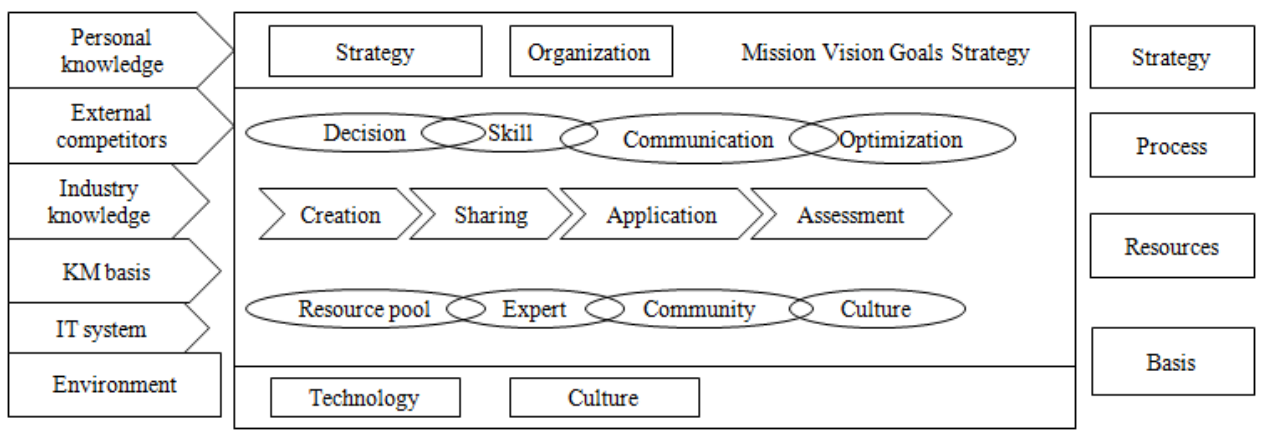

Figure 2. Knowledge Management Framework Model

Source: Wu and Pang (2008), p. 3

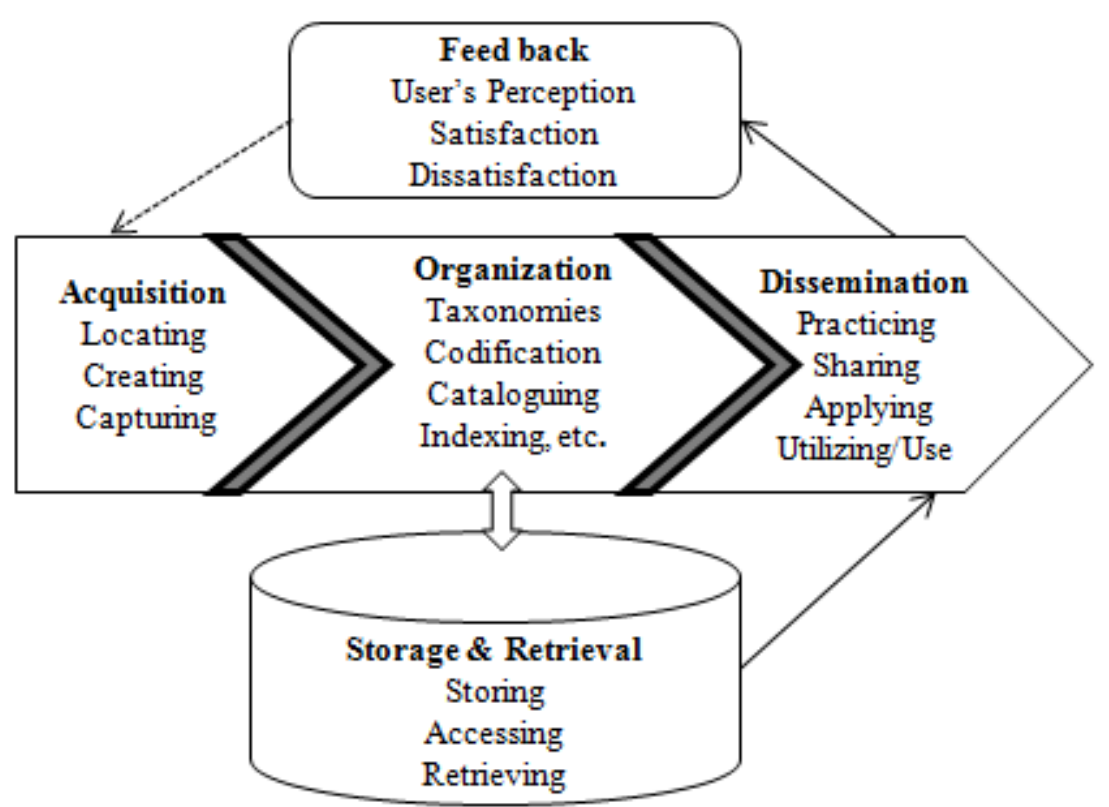

Figure 3. Knowledge Management Process Model

Source: Roknuzzaman, Kanai, and Umemoto (2009), p. 379 
Wu and Pang (2008) developed a knowledge management framework model compatible with a variety of theories, view-points and methods, as can be seen in figure 2 .

From a knowledge management perspective, learners need to go through the process of knowledge collaboration, exchange, sharing, acquisition, creation, distribution, dissemination, storage and personalization in order to acquire knowledge and KM tools assist learners to learn in a ubiquitous learning environment (Lau \& Tsui, 2009). KM techniques can be used to capture, organize and deliver this knowledge and management systems can be used to quickly identify the most relevant information and distribute it to meet specific needs (Sammour et al., 2008). KM is a dynamic and continuous social process (see figure 3) that involves acquisition, organization, storage and retrieval, and dissemination of knowledge resources to user group with relevant feedback to achieve organizational goals (Roknuzzaman, Kanai, \& Umemoto, 2009).

\subsection{Theoretical Framework of the EL System}

Khan (2001) developed a framework for e-learning, which contains eight dimensions: institutional, pedagogical, technological, interface design, evaluation, management, resource support, and ethical (see figure 4), and each dimension has sub-dimensions focusing on particular aspects of the EL environment. This framework can be used to capture an organization's inventory of EL by addressing issues encompassing the eight dimensions of an open and distributed learning environment (Noirid \& Srisa-ard, 2007).

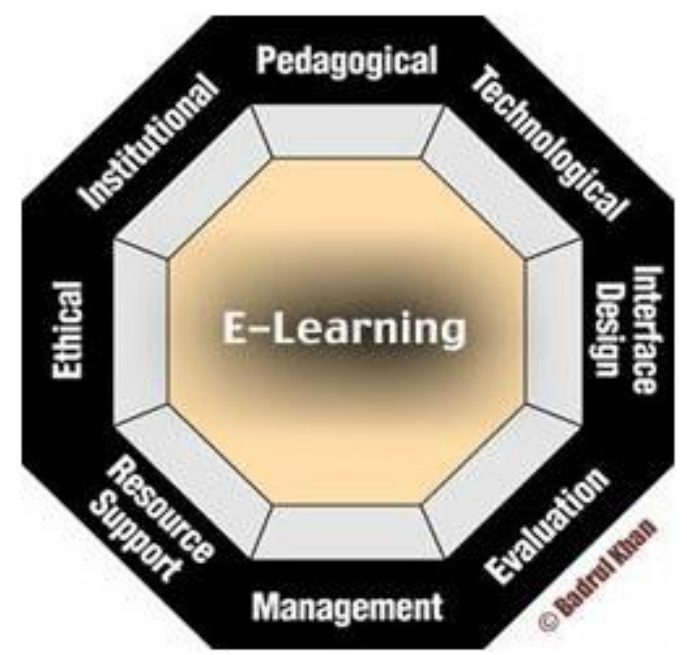

Figure 4. E-learning framework

\section{Source: Khan (2001), P.1}

In addition, Marshall et al. (2003) proposed three types of EL tools: (1) curriculum tools, (2) digital library tools, (3) and knowledge representation-concept maps tools, which emphasize the different parts of the learning process. Curriculum tools provide a systematic and standard environment to support classroom learning; their functions are particularly helpful in the initiation and selection stages. Digital library tools facilitate effective and efficient access to resources to support exploration and collection, while knowledge representation or concept maps tools focus on formulation and representation. Irfan and Shaikh (2008) specified two general categories of learning: 
E-learning by using explicit knowledge and E-learning by using tacit knowledge, as can be seen in figure 5 .

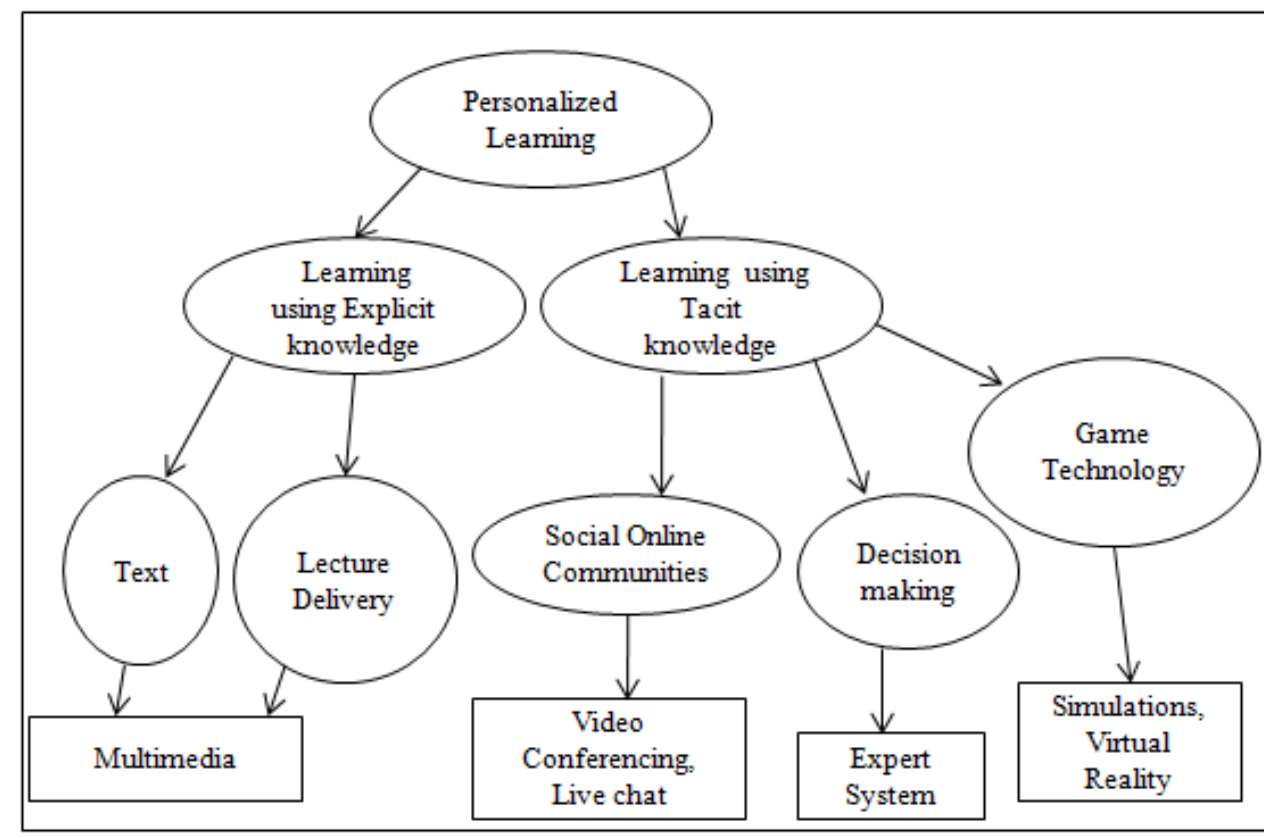

Figure 5. Personalized Learning Model

Source: Irfan and Shaikh (2008), p. 3

\section{Methods}

We used the qualitative approach for this study, and a face-to-face interview of 17 (seventeen) KM and EL research scholars, was conducted to obtain our research objectives. We also carried out this study based on the review of existing concepts and frameworks of KM and EL available in the secondary sources. The interviewees were selected from the participants of the Asia-Pacific Conference on Technology Enhanced Learning (APTEL), on September 24-26, 2010 of Kansai University in Osaka, Japan, and The Fifth International Conference on Knowledge, Information and Creativity Support Systems (KICSS), which took place on 25-27 November, 2010, in Chiang Mai, Thailand. One of the researchers of this study personally attended the above two conferences and initially selected the interviewees based on the titles of their paper about either KM or EL published in the proceedings and program/abstract book, and requested 30 (thirty) participants among the KM and EL research scholars to give a few minutes of their time for the interview during the break of the conference. Seventeen participants were agreed and took part in the interview. Each interview was lasted from 15 to 25 minutes.

The interviews were conducted using a carefully structured, short and open ended questionnaire. The questionnaire was composed of 7 questions pertinent to the background information of the interviewees, 5 questions on KM, particularly about the interviewee's understanding of KM, its main goals, its important components, how the education system can be supported and enhanced by KM, and to what extent KM tools and technologies can be applied in the EL process, and there were 5 questions in 
particular on the interviewee's understanding of EL as well as the main objectives and important components of EL, and how EL systems can support to improve and enhance the learning process. The final question was concerned to the adoption of KM in an EL system. To ensure the anonymity of the interview participants, we categorized the interviewees into three groups: 'Academics' who are directly involved in teaching and research, 'Researchers' who are involved in KM and/or EL research activities, and the 'Practitioners' who are practically working on KM and/or EL in organizations. The results of the study were thematically analyzed and interpreted.

\section{Analysis of Interview Data}

The following data analysis will reflect the relationship between KM and EL through different views and insights of KM and EL research scholars:

\subsection{Attributes of the Interviewees}

Table 1 shows that 11 interviewees were directly involved in teaching and/or research activities of EL and/or KM, holding the position of Professor, Associate Professor and/or Lecturer, while 3 interviewees were involved in doctoral research, and another 3 interviewees were working in an EL or KM unit of other organizations, holding the position titles of EL project officer, IT advisor for EL, or Senior advisor for KM.

Table 1. Attributes of the interviewees

\begin{tabular}{|c|c|c|c|c|}
\hline Academic or administrative position & \multicolumn{2}{|c|}{ Frequency } & Percent & $\begin{array}{l}\text { Cumulative } \\
\text { percent }\end{array}$ \\
\hline \multirow{4}{*}{$\begin{array}{l}\text { Lecturer/Associate Professor/ Professor } \\
\text { (Academics) }\end{array}$} & Professor & 8 & \multirow[t]{4}{*}{64.70} & \multirow[t]{4}{*}{64.70} \\
\hline & $\begin{array}{l}\text { Associate } \\
\text { Professor }\end{array}$ & 2 & & \\
\hline & Lecturer & $\overline{l 1}$ & & \\
\hline & Total & 11 & & \\
\hline $\begin{array}{l}\text { EL and/ or KM doctoral researcher } \\
\text { (Researchers) }\end{array}$ & \multicolumn{2}{|c|}{3} & 17.65 & 82.35 \\
\hline $\begin{array}{l}\text { Information Technology Advisor/ E-learning } \\
\text { project officer/Senior Advisor (Practitioners) }\end{array}$ & \multicolumn{2}{|c|}{3} & 17.65 & 100.00 \\
\hline Grand total & \multicolumn{2}{|c|}{$\mathrm{N}=17$} & 100 & \\
\hline
\end{tabular}

\subsection{Geographical Distribution of the Interviewees}

The geographical distribution of the interviewees is as shown in figure 6 that 9 (nine) interviewees come from Asia, including 4 from Taiwan, 2 from Japan, 1 each from Saudi Arabia, China and Thailand; 2 (two) are from Europe, counting 1 each from Italy and Turkey, 2 (two) are from America, including 1 each from the USA and Canada, 3 (three) are from Oceania, covering 2 from Australia and 1 from New Zealand and 1(one) from Africa (South Africa). 


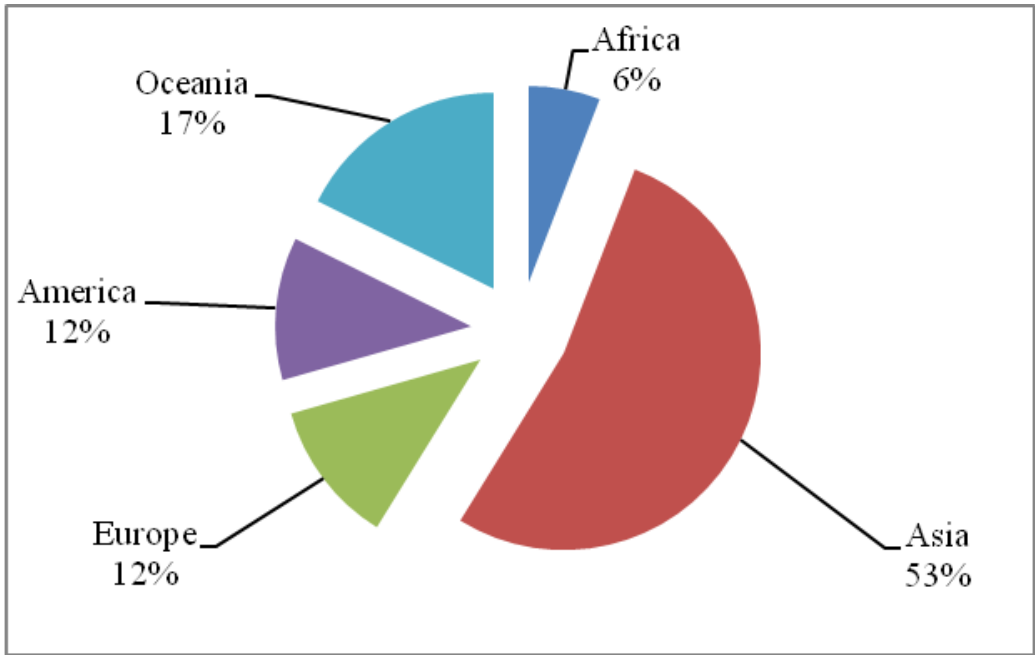

Figure 6. Geographical distribution of the interviewees

\subsection{Understanding of $\mathrm{KM}$}

Different interviewees expressed their understanding of KM from different perspectives although the gist of their understanding was almost the same. For example, one academic reported that KM is still not a clear concept to many. But as far he is known, KM is an advanced and sophisticated process to manage and manipulate, preserve and disseminate knowledge assets, while another academic stated that KM is nothing but the process of creating, acquiring, manipulating, storing and disseminating knowledge. Similarly, another academic asserted that KM is an initiative to capture, manage and disseminate both tangible and intangible knowledge assets available in implicit and explicit knowledge. According to one practitioner, knowledge management can refer to the whole of human experience and the assembling and storage of information prior to and since the advent of information technologies (IT), but in the digital age, it usually refers to computer databases and to how knowledge is stored and accessed, for example a Learning Management System (LMS). Usually, government agencies, educational institutions and business organizations control the access to knowledge. One researcher reported $\mathrm{KM}$ as a way to utilize existing knowledge and information, and to cope with problems by producing new knowledge, and another academic stated that KM consists of strategies and practices used in organizations, to create knowledge, acquire knowledge, store, and disseminate knowledge. According to another academic, KM is a social as well as technological process that facilitates the mechanisms to utilize, harvest, transfer, create and preserve knowledge in both tacit and explicit forms. They include both hard and soft aspects such as technology, people and knowledge-based systems.

\subsection{Understanding of EL}

Most of the interviewees considered EL as a learning and teaching process, although there were some variations in their understanding and description. For example, one academic argued that the other name of online learning is E-learning, while another academic asserted that EL is a process of delivering education through the use of Internet and computer technology. Similarly, another academic stated that EL is about studying professional curricula through the Internet with some kind of pedagogy embedded in the 
learning process. Furthermore, one of the academics reported that any online and computer assisted learning support EL and it could be as comprehensive as a substitute to face-to-face learning or even a supplement to face-to-face learning. According to one practitioner, an E-learning system can be a range of technologies from synchronous (website, email, ubiquitous technologies) to a 'blend' of technologies that include also asynchronous technologies (website, web conferencing, Skype). One academic stated Elearning as a framework to support independent learning using various communication methods that reduce the barrier of distance, while another academic reported that EL is an Internet-based learning/web-based learning. It is interesting to note that one academic described EL as Blackboard software to manage, organize and support education.

\subsection{Main Objectives or Goals of KM}

The objective of $\mathrm{KM}$ is to improve the quality of the contributions people make to their organizations by helping people to make sense of the context within which the organization exists, to take responsibility, to cooperate and share they know and learn, and to effectively challenge, negotiate and learn from others (Chu, Wang, \& Yuen, 2011). However, the interviewees were requested to briefly enumerate the main objectives or goals of KM. The comments of the interviewees regarding the objectives or goals of KM are thematically analyzed below:

\subsubsection{Create, Manipulate and Manage All Types of Knowledge}

One researcher reported the goal of KM is to classify, categorize and organize knowledge, while another academic stated that one of the objectives of KM is to manage knowledge assets. Furthermore, one academic mentioned that the main objective of KM is to create knowledge and to manipulate knowledge, and another academic reported that the goals of KM are to catalogue, preserve and archive. Another researcher explained that the goal of $\mathrm{KM}$ is to collect, share, manage and to enrich knowledge, while one academic reported that the objective of $\mathrm{KM}$ is to produce more knowledge, transfer knowledge and solve problems. On the other hand, another academic stated that the main goal of KM is to maintain and manipulate knowledge resources.

\subsubsection{Sharing, Disseminating and Reusing of Knowledge}

According to one academic, one of the main goals of $\mathrm{KM}$ is to share knowledge in organizations, while another academic explained the goals of KM as identifying, storing and sharing knowledge, and another academic reported that one of the goals of KM is to share knowledge through dissemination. Furthermore, one academic stated that the significant goals of $\mathrm{KM}$ are identifying knowledge assets, capturing, maintaining, retrieving, disseminating, reusing knowledge etc., while one researcher reported that KM helps the distribution of knowledge, and another academic noted that one of the goals of $\mathrm{KM}$ is to support the decision making process through disseminating relevant knowledge. One academic, on the other hand, reported that knowledge sharing and reusing is one of the important objectives of KM. 


\subsubsection{Foster Innovation by Free Access to Knowledge and Enhance the Performance of Human Resources}

One academic reported that one of the objectives of $\mathrm{KM}$ is to foster innovation by providing a free flow of information, while another academic stated that one of the goals of $\mathrm{KM}$ is to workflow efficiently or effectively. Another academic noted that one of the objectives of $\mathrm{KM}$ is to facilitate and provide instructors and learners with easy access and free flow to knowledge assets. According to one academic, one of the significant goals of $\mathrm{KM}$ is to improve and enhance the performance of human resources through appropriate KM streamline operations and to reduce costs by eliminating the redundancy of knowledge resources. Conversely, another researcher reported the goal of KM is to achieve or attain objectives efficiently and effectively by using knowledge.

\subsection{Main Objectives or Goals of EL}

EL involves the use of Internet technology to provide education where the instructor and students are partially or completely geographically dispersed (Adiele \& Nwanze, 2010). The interviewees were asked to indicate the main objectives or goals of EL system. The data and/or information obtained from the interviewees have been analyzed under some pertinent headings:

\subsubsection{Access to Knowledge Assets Easily and Offer Education for All}

According to one academic, the main objectives of EL are to allow learners an easy access to knowledge assets and to support independent learning. On the other hand, one researcher reported that the goal of EL is flexibility, personalization and modularization of learning, and another academic reported that the main objective of EL is to provide education for all irrespective of age, race, abled or disabled people, etc. In addition, one academic reported that the goal of EL is to make education more global and for all, while another academic mentioned that the goal of EL is to provide and accommodate education to the learner's own place, so that one can obtain education staying at home or at the work place. Furthermore, one academic reported that one of the objectives of EL is to reduce the barrier of place and/or person dependence, while another academic noted that the goal of EL is to provide education at any time and anywhere in the world.

\subsubsection{Enhance Learning Experiences Using ICT and Improve the Learning Platform}

According to one academic, enhancing learning experiences using information and communication technology (ICT) is one of the main goals of EL, while another academic reported that the basic objective of EL is to transfer a learner from a role of passively accepting the course content organized by instructors, into another role of proactively selecting the customized courses and contributing their knowledge to continuously improve the learning platform.

\subsubsection{Archive Learning Resources, Manage All Types of Knowledge and Facilitate Searching}

One practitioner reported that the main objectives of EL are to archive the learning objects and to manage both explicit and tacit knowledge. Another goal of EL system is to 
manage the users as well. According to one researcher, the main goals of an EL system are to facilitate searching, forums announcements, uploading class lectures, etc.

\subsection{Significant Components of KM}

We requested the interviewees to specify the important components of KM. The data, obtained from the interview, are summarized in figure 7, and are analyzed under the following specific headings:

\subsubsection{Suitable Infrastructure/Technological Support with Modern ICT}

A strong technological infrastructure is a prerequisite for implementing KM successfully (Sammour et al., 2008). One academic of the interviewees reported similarly that one of the most important components of $\mathrm{KM}$ is the sufficient technological supports with modern ICT, while another two academics identified technology or information technology to facilitate communication/exchanges as the significant components of KM. In this regard, one practitioner noted that the SECI (Socialization, Externalization, Combination, Internalization)-the knowledge creation process and Ba (Japanese concept, which roughly means 'place' (Nonaka \& Konno, 1998))-base for knowledge creation is the major components of KM, while one researcher stated that the significant components of $\mathrm{KM}$ are to attain ways to produce ideas, to solve problems.

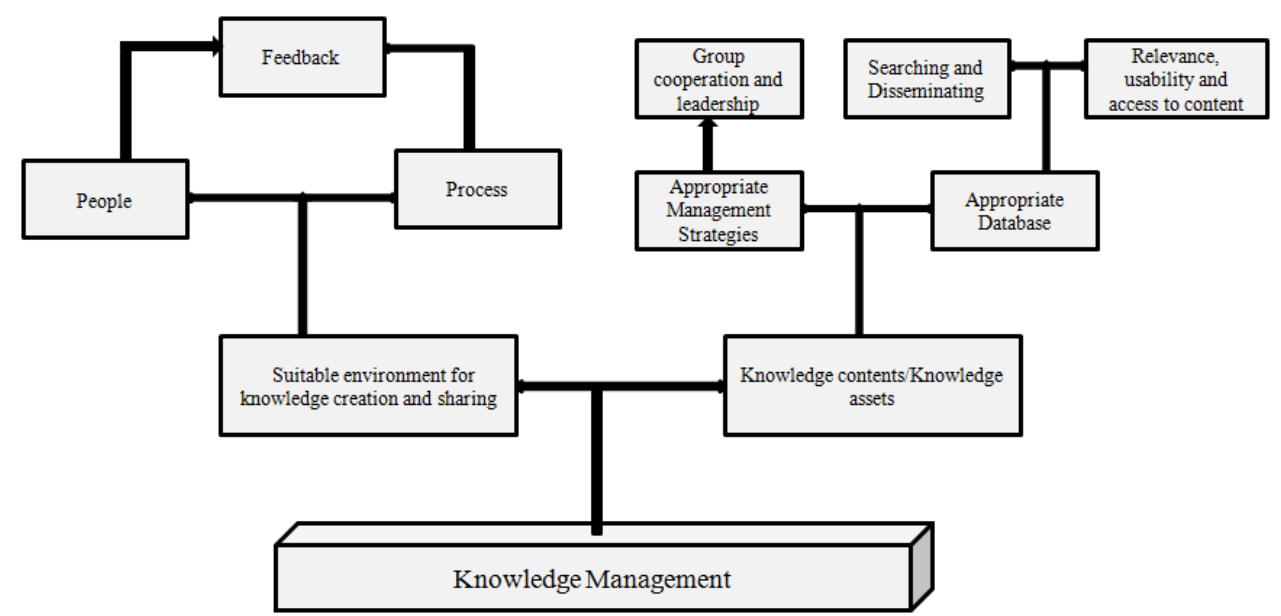

Figure 7. Significant components of Knowledge Management

\subsubsection{Process, People, Feedback and Group Cooperation}

According to one academic, the significant component of $\mathrm{KM}$ is the social process to empower knowledge user, while another two academics identified process, particularly the business process as one of the important components of KM. One academic considered the people, i.e. knowledge engineers, users, etc as the important components of KM, while another two academics reported feedback and group cooperation are the significant components of KM, and one practitioner noted that leadership is one of the major components of KM. 


\subsubsection{Knowledge Contents or Assets}

One academic identified knowledge contents, both tacit and explicit, as one of the significant components of $\mathrm{KM}$, while another academic considered the content of the body of knowledge as one of the important components of KM. On the other hand, two academics stated content authoring, administration, physical materials as well as computer files and handouts are the significant components of KM, while another researcher noted that tacit and explicit knowledge, are major components of KM.

\subsubsection{Appropriate Database and Management Strategies}

One academic considered the database system and/or the data warehouse as important components of KM, while another academic reported that an appropriate database is a major component of KM. Similarly, one academic stated that database technology and knowledge management tools are the significant components of KM, while another academic reported that appropriate knowledge management strategies are vital components of KM.

\subsubsection{Relevance, Usability, Searching and Disseminating}

One researcher described classifying, organizing, use and reuse of knowledge as major components of $\mathrm{KM}$, while another practitioner reported that relevance, usability and access to content are the important components of KM. One academic reported taxonomy, and another academic noted reusability of information as significant components of KM. One practitioner, on the other hand, stated that quick retrieval of information is one of the major components of $\mathrm{KM}$, while another academic reported searching/disseminating as important components of KM. Furthermore, one researcher stated that the significant components of $\mathrm{KM}$ are to retrieve existing information and knowledge from a memory or database.

\subsection{Significant Components of an EL System}

Based on the interview data, the significant components of an EL system are illustrated in figure 8 and the insights and views of the interview participants are explained under some specific relevant headings:

\subsubsection{People (Instructors/Teachers or Learners/Students) and ICT Experts}

One academic reported that instructors who establish physical contact are important components of EL systems. Similarly, another two academics noted that instructors/teachers as well as learners/students are the most significant components of an EL system. Another academic identified learners/students with minimum technical skills as one of the important components of an EL system, while one practitioner considered facilitators, IT administrators and instructors as major components of EL system. Furthermore, another practitioner noted that personnel with experience in the adaptation of content for e-learning are vital components of EL system, while one academic mentioned that an EL system is composed of learners/students with minimum ICT knowledge skills and instructors/teachers with standard ICT knowledge skills. 


\subsubsection{Technical Infrastructure and Suitable Environment}

According one academic, technical facilities equipped with computers and software are one of the significant components of an EL system. One researcher described different interfaces for users (instructors and students) as major components of EL system, while another academic reported that software, a computer network and computers are the most important components of an EL system. Furthermore, another academic reported that proper technological infrastructures as well as a good learning environment for learning and teaching are major components of an EL system.

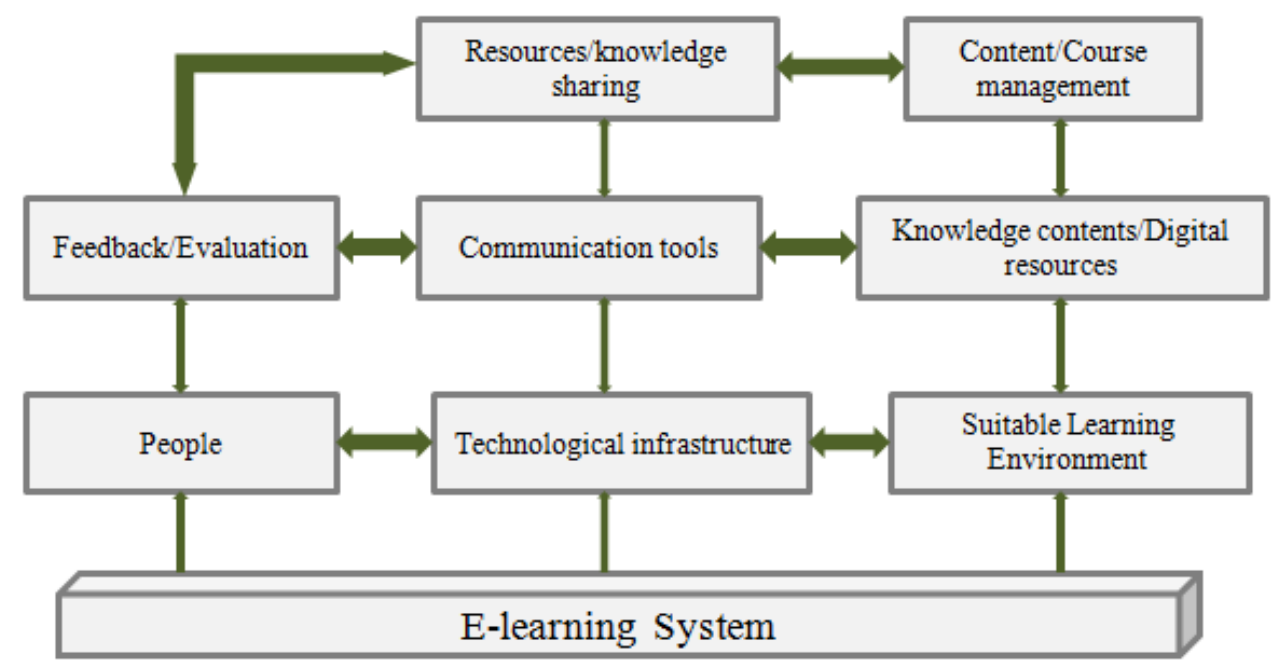

Figure 8. Significant components of an E-learning system

\subsubsection{Communication Tools and Knowledge or Resource Sharing}

One academic stated that communication tools such as the internet and a network of learners and instructors who exchange information are significant components of an EL system. Another three academics reported that high speed of Internet or Intranet, as well as the computer and internet connection are significant components of EL systems. Another academic, on the other hand, reported that resources and/or knowledge sharing is one of the significant components of an EL system.

\subsubsection{Knowledge Content or Digital Resources and Course Management}

According to one academic, e-books, video and audio files are major components of an EL system. Another two academics stated that digital contents/resources as well as content capture, shape, upload and share of the contents are major components of an EL system, while one researcher identified contents of the information sources as significant components of an EL system. Furthermore, one academic reported that content development, content management and course/enrolment management are the significant components of EL systems, while another academic also reported content management as one of the important components of an EL system. 


\subsubsection{Feedback or Evaluation}

One academic reported that an evaluation and/or monitoring system is one of the important components of an EL system to ensure that the system is effective, while another academic stated that progress tracking, including assessment and feedback, etc. are significant components of an EL system.

\subsection{The Education System Supported and Enhanced Using KM}

KM involves creating and organizing a university's knowledge, sharing that knowledge with appropriate people and groups, and then facilitating the application of that knowledge to achieve university missions and goals (Geng et al., 2004). According to one academic, KM can support and enhance an education system through the acquisition, organization, storage, retrieval and dissemination of knowledge assets, while another academic reported that, through appropriate and proper management of knowledge resources, an education system can be supported and enhanced by KM, for instance, if the knowledge contents or knowledge resources are organized and well-managed, education can be delivered easily, accurately and timely. Furthermore, one academic stated that teaching and learning is a process that highly relies on the externalization of knowledge, creating new knowledge, transferring knowledge from the teacher to the students and also from students to students or to teachers, while another academic expressed the opinions that an education system can be supported and enhanced by creating a model of $\mathrm{KM}$ in the education system, designing technical systems and personnel information literacy. One practitioner reported that an education system can be supported and enhanced by using $\mathrm{KM}$ as a repository for curriculum and resources (content). One academic expressed his views as education system can be supported and enhanced by facilitating information dissemination, and another academic reported that information searching and teaching/sharing of information can support and enhance an education system. In addition, another academic stated that KM can support the learning process by providing necessary knowledge resources to the teachers/instructors as well as learners/students. It can also help those giving directions to access appropriate knowledge/information.

\subsection{EL Systems Support to Improve and Enhance the Learning Process}

EL as a new educational paradigm refers to utilization of web-based technologies to deliver learning activities that enhance knowledge and performance (Zhan \& Chang, 2008). Called Course Management Systems (CMS) or Learning Management Systems (LMS), Web-based Systems allow instructors and students to share instructional materials, make class announcements, submit and return course assignments, and communicate with each other online (Lonn \& Teasley, 2009). One academic of the interviewees reported that EL systems can help learners to be equipped with technical knowledge or skills. They also enable the whole learning process to be more interactive, in improving and enhancing the learning process. Another academic stated reducing the time and physical barrier in retrieving information that supports the formation of new knowledge and increasing the curiosity for new knowledge through easier access to information. Furthermore, one academic noted that learning anytime/anywhere and sharing information could be enjoyable and fun to improve and enhance the learning process, while another academic stated that EL systems support to improve and enhance the learning process by making learning easy and interactive. According to one practitioner-

"I believe e-learning systems are revolutionizing education and all areas of temporary life. The 'anywhere, anytime' dynamic that e-learning makes possible 
is enabling all learners to access information and thus is enhancing and impacting the lives of entire communities."

One researcher, on the other hand, expressed her opinions in this regard, that EL is more convenient for handling assignment, but for discussion, we use instant messaging or social networking more, and one practitioner reported that EL systems support to improve and enhance the learning process by easy access, to access more resources, customizable allow, meeting individual needs, and inexpensive or free access to the knowledge assets (although the initial cost would be high). According to one academic, there are many ways to enhance learning- he often uses animation, visualization, simulation, etc., to explain complex scientific and mathematical topic. It gives better experiences to the learners. Similarly, another academic stated that, through engagement, interaction, visual effects and communication an EL system supports to improve and enhance the learning process. According to another academic, in a traditional learning system, some students are afraid of asking questions to their teachers. But in a synchronous EL system, students can ask questions easily and obtain a reply instantly. In addition, EL systems make the learning process easier and simpler for the learners.

\subsection{KM Tools and Technologies Applied in the EL Process}

KM tools can be categorized into four groups: tools that support knowledge sharing (e.g. groupware, intranets and the Internet); tools that support knowledge distribution (e.g. electronic calendars, desktop database, desktop publishing); tools that support knowledge capture and codification (e.g. expert systems, neural networks and intelligent agents); and tools that support knowledge creation (investment workstation, CAD, and virtual reality) (Laudon \& Laudon, 1999). One practitioner stated that KM tools and technologies can be applied in the EL process as a place to store course content (asynchronous technologies), and thus a place where teachers and learners can access the course content, while one researcher noted that KM tools and technologies can be applied in social bookmarking and social tagging. According to one academic, most of the KM technologies and tools can be adapted to teaching and the learning process. KM success depends on organizational culture and people's behaviour. In his opinion, face-to-face interaction and group support in e-learning are hard to implement. KM tools and techniques may be of help. Furthermore, two academics reported that database technology and some ontology of KM can be used and applied in the EL process, and KM tools and technologies can be applied to disseminate and share knowledge, to store and retrieve knowledge using KM database technologies. One researcher stated that KM tools and technologies can be applied to assist one to produce new ideas or new knowledge, and another practitioner reported that one can cover explicit knowledge by using KM tools and technologies. In addition, another academic stated that-

"KM tools and technologies can be applied in the EL process as follows: KM database technologies can be used in the EL process; KM ontology can also be used in the EL process; KM planning and management strategies can easily be applied in the EL process".

\subsection{Insights and Views of the Interviewees Regarding the Adoption of KM in an EL System}

One practitioner reported that she sees the two as being linked- KM is the repository of the content that teachers and learners access using an EL system, while one academic stated that adoption of KM in an EL system would bring good results to both KM and EL. It is a recognized fact that there are some common tools and technologies of KM and EL, and if they can exchange, adopt and embrace those tools and techniques both KM and EL 
will have adequate benefits and advantages. Very similarly, one academic argued that the adoption of KM in an EL system could significantly contribute to the enhancement of the learning process. Particularly, an EL system should adopt the most essential and necessary components of KM that will enhance the performance of EL. Conversely, another academic stated that EL can support KM by enabling the exchange of ideas and knowledge regardless of physical and temporal barrier. However, it still requires trust and positive relationships between the users to be established, perhaps through an actual physical encounter prior to the use of technology to continue the process. Furthermore, another academic stated that-

"Yes, I think, the adoption of KM in an EL system must affect positively to the whole learning process. As both KM and EL are closely related and they can share some tools and technologies with each other for their mutual benefits and advantages".

On the other hand, one researcher reported that probably, using KM to integrate similar subject matters or knowledge is helpful to education. Again, one academic stated that it seemed to him that EL should adopt some components, tools and technologies of KM, which will significantly enhance the performance and quality of EL. This can be accomplished through using KM and EL simultaneously, or sharing and exchanging with each other. Another researcher stated that KM plays an important role in an EL system in meeting user requirements.

\section{Common Goals and Significant Overlaps of KM and EL}

\subsection{Common Goals of KM and EL}

Many corporations are discovering that EL has many of the same attributes as basic KM processes, and thus can be a tool for KM (Wild, Griggs, \& Downing, 2002). However, based on the views of both KM and EL research scholars, we discovered some of the strategic common goals of both KM and EL described below, which were also supported by the related existing literature:

\subsubsection{Establish Collaborative Learning Environment and Practice a Co- operative Culture}

The most common goal of both $\mathrm{KM}$ and EL is to establish a collaborative learning environment and practice a co-operative culture for knowledge acquisition and sharing. A major aim of KM is to establish a positive learning environment in which people can conduct all sorts of learning activities and share knowledge with other people in organization (Bukowitz \& Williams, 1992; Hong \& Kuo, 1999; Martensson, 2000). For instance, one academic stated that the significant component of KM is the social process to empower knowledge user and another academic reported that group cooperation is the significant components of KM. Simultaneously, another academic stated that the significant goal of EL is to offer education for all walks of life and to deliver education promptly to any parts of the world.

\subsubsection{Develop a Technological Infrastructure}

The significant common goal of $\mathrm{KM}$ and EL is to create a suitable technological infrastructure for acquiring, organizing, storing, manipulating, maintaining and disseminating knowledge as well as ensuring its best utilization. For example, one academic stated that the suitable environment for knowledge creation and sharing as well as knowledge users is the important components of KM, while another academic reported 
that proper technological infrastructures as well as a good learning environment for learning and teaching are major components of an EL system.

\subsubsection{Allow Users to Access Knowledge Assets}

Another common goal of KM and EL is to allow learners and /or users to access knowledge resources so that they can obtain knowledge and receive education easily. For instance, one academic stated that one of the objectives of KM is to facilitate and provide instructors and learners with easy access and free flow to knowledge assets, while one practitioner stated that the objective of EL is to use digital technologies to enable learners to access educational resources from anywhere and at any time.

\subsubsection{Manage Tacit and Explicit Knowledge Contents}

The objectives of both KM and EL are to manage both the tacit and explicit contents of knowledge for their appropriate delivery and/or dissemination among the learners or users. For example, one academic stated that the main goal of KM is to maintain and manipulate knowledge resources, while another practitioner reported that the main objectives of EL are to archive the learning objects and to manage both explicit and tacit knowledge.

\subsection{Significant Overlaps between $K M$ and EL}

By reviewing the existing literature as well as data and/or information obtained from the interviewees, we observe that there is a close relationship and significant overlaps between EL and KM. For instance, Liu and Wang (2009) argued that EL is the technology and tool supporting KM, and KM is the premise and operational platform of EL systems (see figure 9).

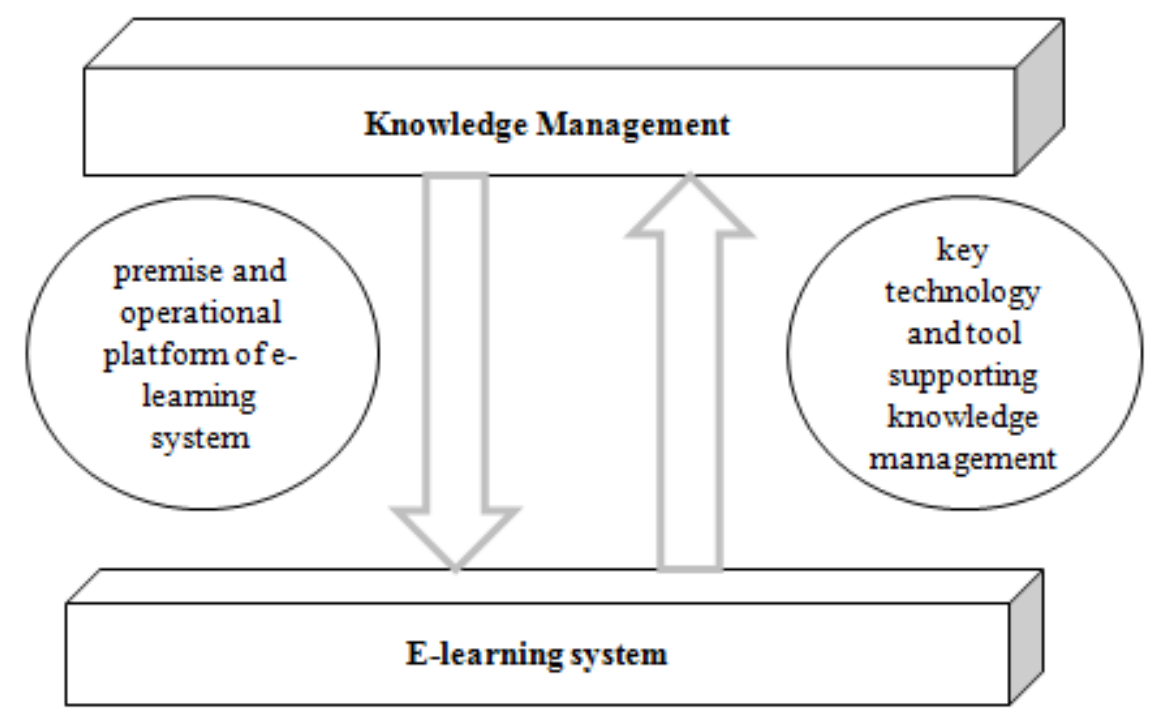

Figure 9. Relationship between KM and the EL system

Source: Liu and Wang (2009), P. 330

However, we identified some of the significant overlapping areas between EL and $\mathrm{KM}$ from existing concepts and literature, as well as views from the interviewees where EL system and KM have the opportunity to share and contribute to each other (see figure 10). 


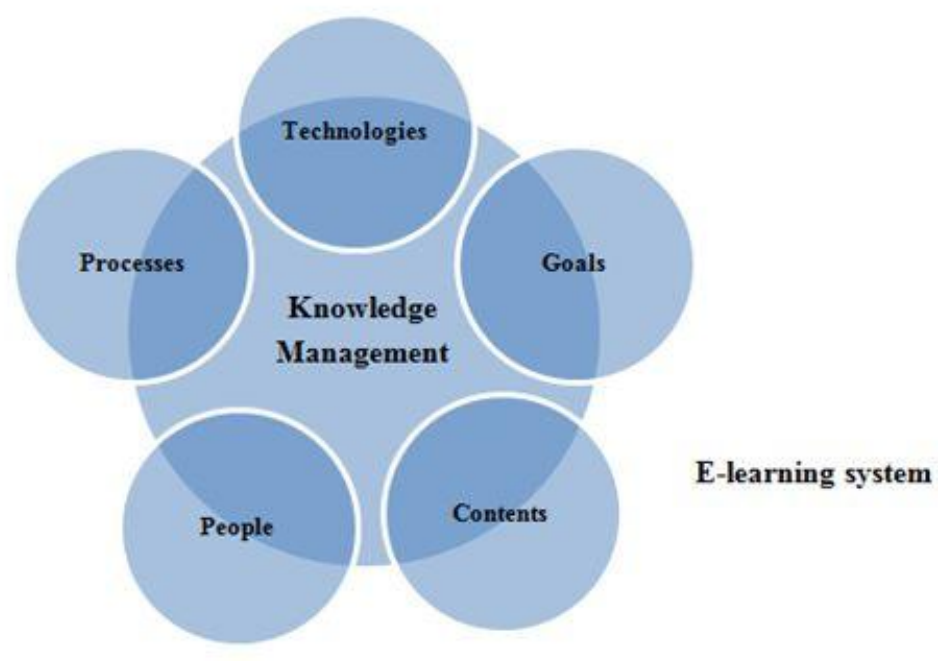

Figure 10. Significant overlaps between an EL system and KM

\subsubsection{Goals}

The basic goal of both KM and an EL system is the same, that is, to allow learners and/or users to access knowledge resources, and their aim is to establish a suitable, collaborative and convenient learning environment through which learners and/or users can access and acquire knowledge easily. Both EL and KM aim at improving individual, team and organizational performance through knowledge dissemination (Ponce, 2003). In addition, one researcher of the interviewees reported that she thought that KM and EL are same in some context.

\subsubsection{Technologies}

The Internet and information and communication technologies (ICTs) are the backbone of EL, while KM also needs technological support. Without the support of ICTs, such as digital repositories, search engines, Internet, intranets, extranets, document management systems, multimedia, etc., both the KM and an EL system cannot work properly or, in some cases, they do not work at all. KM and EL both address the same fundamental problems: facilitating learning in organizations (Schmidt, 2005). Interestingly, one academic reported that KM tools and technologies can be applied in all aspects of EL, starting from course design until assessment and evaluation.

\subsubsection{Processes}

Both EL and KM are involved in all the processes of knowledge acquisition, organization, storage, retrieval, sharing, application, innovation, dissemination, etc. EL can be abstracted as the process in which staff makes use of computer and Internet resources to learn to acquire knowledge and to apply knowledge, while the essence of KM is the process for people to explain a lot of data, and information with science and technology media (Chunhua, 2008). It is interesting to note that one academic expressed his views very positively, that really EL systems are tremendously supporting through their various 
user friendly tools to improve and enhance the learning process, while another academic stated that KM planning and management strategies can easily be applied in the EL process".

\subsubsection{People}

People are the key resources for both the KM and EL systems, which require personnel or instructors as well as users or learners with great enthusiasm and initiative. People with appropriate ICT, interpersonal communication, managerial, behavioural and professional skill, can play a vital role in designing, operating, managing and maintaining the KM and EL systems. For instance, one academic stated that the expert professionals are one of the significant components of KM, while another academic reported that ICT experts or qualified who are sufficiently equipped with ICT skills are essential components of an EL system.

\subsubsection{Contents}

Acquisition, management, sharing and dissemination of data, information and knowledge are the basic and similar functions of KM and an EL system. Both KM and an EL system focus on transferring, distributing and delivering explicit and tacit content of knowledge resources to an individual learner to group or organizations and vice versa. For example, one academic stated that KM can support the learning process by providing necessary knowledge resources to the teachers/instructors as well as learners/students. It can also help those giving directions to access appropriate knowledge or information.

\section{The Theoretical Model for Adopting KM and EL}

The term 'adopt' has been used here as the meaning of, 'to start to use a particular method'. Although EL can be offered using tacit knowledge (see figure 5) and explicit knowledge, it can adopt the conversion method of KM to convert tacit knowledge into explicit knowledge to become a more effective learning system. Therefore, we propose the following model for adopting KM in EL through the conversion of tacit to explicit knowledge (see figure 11). In this model, knowledge creation, acquisition, evaluation and feedback have been presented as a part of tacit knowledge and their conversion into explicit knowledge. Conversely knowledge organization, storage, dissemination and retrieval have been illustrated as explicit knowledge, which can be converted into tacit knowledge as well.

\subsection{Knowledge Creation and Acquisition}

Knowledge creation can be defined as the process of developing new knowledge from data, information, prior knowledge and experience (Sabherwal \& Sabherwal, 2005). In the first stage of the model as shown in figure 11, tacit knowledge is created through direct interactions (Human-Human) or online interactions (Human-Machine) through live chat or video conferencing, etc., among the learners/users and instructors/managers, and converting tacit into explicit knowledge in the KM and the EL environment is performed accordingly. The process of acquiring knowledge is largely supported through direct interaction with suppliers and customers (Nonaka \& Konno, 1998). Therefore, acquisition of knowledge is an important task of both KM and EL, which includes the activities of acquiring both tacit and explicit knowledge resources and capturing them 
from various sources e.g. online chatting, books, journals, reports, manuals, theses, dissertations, conference proceedings, etc. Knowledge in KM and EL can also be acquired from different online sources such as online databases, and from various electronic publications on the Internet.

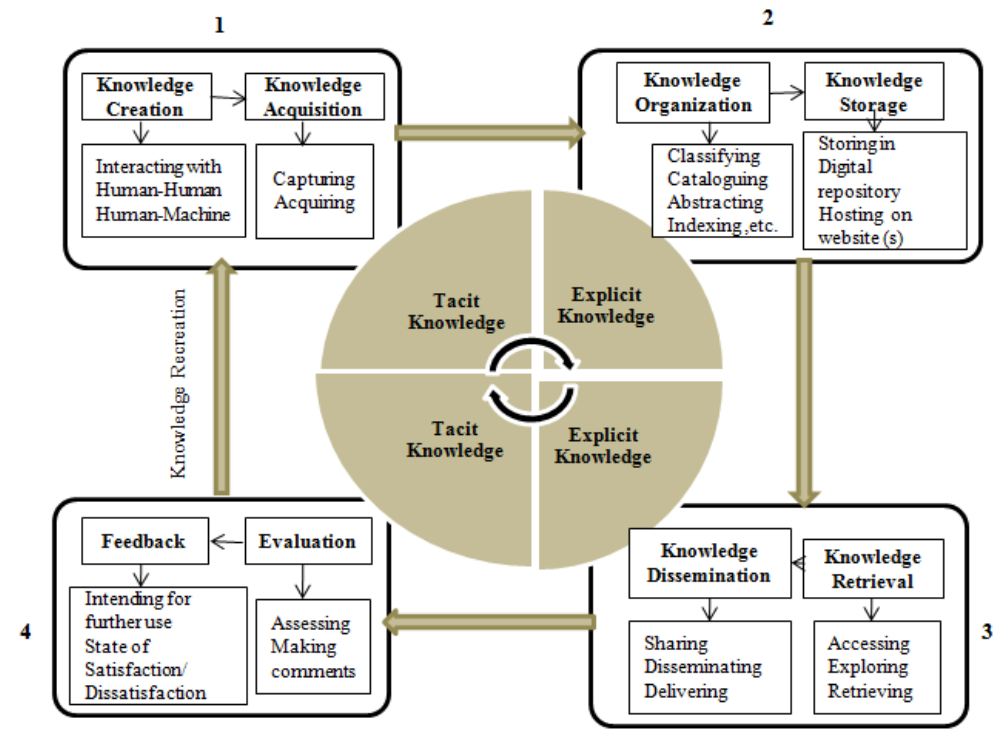

Figure 11. KM and EL Adoption Model

\subsection{Knowledge Organization and Storage}

The organization of knowledge for exploitation and re-use in the modern enterprise is often a most perplexing challenge, and the entire knowledge management life-cycle (for example - create, capture, organize, store, search, and transfer) is impacted by the organization of intellectual capital into a corporate taxonomy or knowledge map (often used interchangeably) (Sharma, et al., 2010). The EL system, on the other hand, organizes its knowledge resources in a suitable and convenient deliverable format so that instructors and/or learners can access and use the resources very easily. Therefore, the model in the second stage shows that EL can adopt some knowledge organization and storage techniques of KM to enhance the learning process. The retrieval process highly depends on the proper storage of knowledge in both the KM and the EL system. Hence systematic and well organized knowledge resources are stored in a digital knowledge repository or hosted on websites for their preservation, their appropriate and multiple uses in both the KM and the EL environment.

\subsection{Knowledge Retrieval and Dissemination}

To facilitate accessing, exploring and retrieving knowledge resources, a good number of tools and techniques, such as browsing, searching, data mining, metadata, knowledge mapping, etc., are being used in KM. In the third stage of the model, it is suggested that these types of tools may be adopted in EL for structuring and retrieving explicit digital knowledge resources. Ponce (2003) argued that data mining methods can be used in EL systems to "detect unknown patterns in user learning behaviour, learning resources and knowledge mastering bottlenecks". 
Knowledge dissemination enables not only knowledge of the organization to be widely spread amongst its members, but also to the public who need it to obtain access to the services or products provided by the organization ( Zhang \& Kim, 2011). The model also reveals in this stage that both KM and EL use email, chat rooms, discussion forums, video-conferencing and other tools of $\mathrm{KM}$ as well as EL, and social networks tools for the purpose of knowledge sharing, disseminating and delivering of knowledge.

\subsection{Evaluation and Feedback}

Assessment or responses from the learners/users or instructors in the form of comments or suggestions regarding instruction and the EL environment are very much effective for solving the existing problems of the EL system. Hence, user evaluation is treated as the most significant part of the EL system. This model demonstrates the evaluation and feedback in its fourth stage and suggests that KM can adopt the evaluation method together with its feedback process. Feedback loops aimed at supporting knowledge maintenance and forgetting can greatly assist efforts to ensure that organizational knowledge remains relevant and does not become overwhelmed by obsolete knowledge (Nevo, Furneauz, \& Wand, 2008). Feedback from the users can be received through email or in web-based electronic form. Depending on the user's perception regarding satisfaction or dissatisfaction or intention to continual use of the system, both the KM and an EL system can be refined, readjusted or redesigned for the enhancement of their performance.

\section{Conclusions and Implications}

The main goal of the study was to obtain the insights and views from KM and EL research scholars through a face-to-face interview for determining the relationship between KM and EL as well as for adopting KM in an EL system. The insights and views of the KM and EL research scholars indicate that there is a close relationship between $\mathrm{KM}$ and EL in terms of their goals or objectives, components, tools and technologies, etc. for instance, establishing a collaborative learning environment and practicing a cooperative culture for knowledge acquisition and sharing; creating a suitable technological infrastructure for acquiring, organizing, storing, manipulating, maintaining and disseminating knowledge; allowing learners and/or users to access knowledge resources; and finally managing both tacit and explicit knowledge, have been identified as common goals or objectives of both KM and EL. We also explored that both of them have some significant overlapping areas such as technologies, goals, processes, people and contents and some opportunities for mutual sharing, exchanging and adopting e.g. one academic stated that as both KM and EL are closely related and they can share some tools and technologies with each other for their mutual benefits and advantages, while another academic reported that KM tools and technologies can be applied in all aspects of EL, starting from course design until assessment and evaluation.. In fact, learning is a process of interaction between learners, instructor, learning content, and learning peers. Its effectiveness depends on the success of closely interplay among these elements (Tai \& Ting, 2007). We proposed a KM and EL adoption model, which could significantly contribute to utilizing these elements properly for the enhancement of EL performance. We identified the conversion of tacit knowledge, the promotion of knowledge organization and retrieval, the enhancement of knowledge sharing, and the proper management of knowledge resources as the implications and expected benefits through the adoption of KM and EL. However, we selected the interviewees from two 
international conferences held in 2010 and conducted the interviews to only $17 \mathrm{KM}$ and EL research scholars worldwide, and therefore the results of this study may not reflect the general scenario of the world regarding the determination the relationship between KM and EL, and the adoption of KM to an EL system.

We built a model based on existing concepts and the views and insights of KM and EL research scholars worldwide. Basically it was a model building study; therefore the model has not been verified or tested. However, we can expect the following implications and benefits through the KM and EL adoption model:

\subsection{Conversion of Tacit Knowledge}

Tacit knowledge can be communicated through interaction, collaboration and conversations in communities/network of practices (Roknuzzaman, Kanai, \& Umemoto, 2009). Through the conversion of tacit knowledge into explicit knowledge, the EL system and/or instructor can deliver knowledge resources very easily, and users/learners can acquire knowledge more conveniently.

\subsection{Promotion of Knowledge Organization and Retrieval}

EL can apply and utilize some significant knowledge organization and retrieval techniques and tools of KM through the adoption of KM and EL, which certainly promote the effectiveness of knowledge organization and retrieval of the EL system as well as its learning process. For instance, one practitioner reported that KM is the repository of the content that teachers and learners access using an EL system.

\subsection{Proper Management of Knowledge Resources and Enhancement of Sharing}

EL can utilize the management techniques of KM for the appropriate management of its knowledge resources, e.g., one practitioner reported that KM practices are inherent to socialization, interaction and sharing the teaching and learning environment should target to adopt good KM practices in the right context. Knowledge sharing is seen as central concept of KM, which focuses more attention on tacit knowledge. Therefore, through the adoption of KM to an EL system, EL will be equipped with an opportunity to enhance a collaborative, innovative, open and distributed knowledge sharing culture.

\section{Acknowledgements}

This study is a part of the first author's doctoral research, and is funded by a Scholarship of Ministry of Education, Culture, Sports, Science and Technology (Monbukagakusho), at the Japan Advanced Institute of Science and Technology (JAIST), in Japan. Therefore, we hereby acknowledge the financial support of the Monbukagakusho. We express our deepest thanks to Dr. Jean-Christophe Terrillon, Associate Professor, in the Global Communication Center of JAIST for his assistance regarding copy editing of the article. We also thank the anonymous peer-reviewers for their thoughtful and useful comments, which helped greatly in improving this article. 


\section{References}

1. Adiele, C., \& Nwanze, E.D. (2010). The Dynamics of Interactivity Modeling for eLearning. Knowledge Management \& E-Learning: An International Journal, 2(4), 370-382.

2. Bukowitz, W.R., \& Williams, R.L. (1999). The Knowledge Management Fieldbook. London: Prentice Hall.

3. Chu, K.W., Wang, M., \& Yurn, A.H.K. (2011). Implementing Knowledge Management in School Environment: Teacher's Perception. Knowlege Management \& E-Learning: An International Journal, 3(2), 139-152.

4. Chunhua, Z. (2008). E-learning: The New Approach for Knowledge Management $(K M)$. Retrieved from http://ieeexplore.ieee.org/stamp/stamp.jsp?tp=\&arnumber=4722899.

5. Geng, Q., Townley, C., Huang, K., \& Zhang, J. (2005). Comparative Knowledge Management: A Pilot Study of Chinese and American Universities. Journal of the American Society for Information Science and Technology, 56 (10), 1031-1044.

6. Irfan, R., \& Shaikh, M.U. (2008). Framework for Embedding Tacit Knowledge in Pedagogical Model to Enhance E-learning. Retrieved from http://ieeexplore.ieee.org/stamp.jsp?tp=\&arnumber=4689102/.

7. Khan, B.H. (2001). A Framework for E-learning. Retrieved from http://bookstoread.com/framework.

8. Hong, J.C., \& Kuo, C.L. (1999). Knowledge Management in the Learning Organization. The Leadership and Organizational Development Journal, 20, 207215.

9. Lau, A., \& Tsui, E. (2009). Knowledge Management Perspective on E-learning Effectiveness. Knowledge-Based Systems, 22, 324-325.

10. Laudon, K.C., \& Laudon, J. C. (1999). Management Information Systems: Organization and technology in Networked Enterprise (6th ed.). Prentice Hall, Englewood Cliffs, NJ.

11. Liu, Y., \& Wang, H. (2009). A Comparative Study on E-learning Technologies and Products: from the East to the West. Systems Research and Behavioral Science, 26, 191-209.

12. Lonn, S., \& Teasley, S.D. (2009). Saving Time or Innovating Practice: Investigating Perceptions and Uses of Learning Management Systems. Computers \& Education, 53, 686-694.

13. Lytras, M.D., Naeve, A., \& Pouloudi, A. (2005). Knowledge Management Roadmap for E-learning: the Way Ahead. International Journal of Distance Education technologies, 3(2), 68-75.

14. Marshall, B., Zhang, Y., Chen, H., Lally, A., Shen, R., Fox, E. \& Cassel, L. N. (2003). Convergence of Knowledge Management and E-learning: the GetSmart Experience. Retrieved from http://delivery.acm.org/10.1145/830000/827161/p135marshall.pdf?key1=827161\&key2=4017403721\&coll=GUIDE\&dl=GUIDE\&CFI $\mathrm{D}=89142264 \&$ CFTOKEN $=73110503$.

15. Martensson, M. (2000). A Critical Review of Knowledge Management as a Tool. Journal of Knowledge Management, 4, 204-216.

16. Morales, E. (2005). Knowledge Management for E-learning Based on Learning Objects: A Qualitative Focus. Proceedings of the ITHET 6th Annual International Conference, session 64B. Retrieved from. 
http://ieeexplore.ieee.org/stamp/stamp.jsp?tp=\&arnumber=1560305\&userType $=$ ins t.

17. Nevo, D., Furneaux, B., \& Wand, Y. (2008). Towards an Evaluation Framework for Knowledge Management Systems. Information Technology and Management, 9 (4), 233-249.

18. Noirid, S., \& Srisa-ard, B. (2007). E-learning Models: A Review of Literature. Proceedings of The 1st International Conference on education reform 2007, November 9-11, Mahasarakham University, Thailand. Retrieved from http://www.icer2007.msu.ac.th/paper/ICT1.pdf.

19. Nonaka, I., \& Konno, N. (1998). The Concept of "Ba": Building a Foundation for Knowledge Creation. California Management Review, 40(3), 40-54.

20. Ponce, D. (2003). What can E-learning Learn from Knowledge Management? Paper presented in 3rd European Knowledge Management Summer School, 7-12, San Sebastian, Spain. Retrieved from http://www.knowledgeboard.com/download/720/kmss03_09.pdf/.

21. Roknuzzaman, M., Kanai, H., \& Umemoto, K. (2009). Integration of Knowledge Management Process into Digital Library System: A Theoretical Perspective. Library Review, 58(5), 372-386.

22. Sabherwal, R., \& Sabherwal, S. (2005). Knowledge Management Using Information Technology: Determinants of Impact on Firm Value. Decision Sciences, 36(4), 531-567.

23. Sammour, G., Schreurs, J., AI-Zoubi, A. Y., \& Vanhoof, K. (2008). The Role of Knowledge Management and E-learning in Professional Development. International Journal of Knowledge and Learning, 5, 465-477.

24. Schmidt, A. (2005). Bridging the Gap between Knowledge Management and Elearning with Context-Aware Corporate Learning. Retrieved from http://publications.andreas.schmidt.name/Schmidt_LOKMOL05_extended.pdf.

25. Sharma, R. S., Chia, M., Choo, V., \& Samuel, E. (2010). Using Taxonomy for Knowledge Audits: Some Fields Experiences. Journal of Knowledge Management, 11(1), Retrieved from http://www.tlainc.com/articl214.htm.

26. Tai, Y., \& Ting, R.Y-L. (2007). Authoring Tools in E-learning: A Case Study. Proceedings of Seventh IEEE International Conference on Advanced Learning Technologies (ICALT, 2007). Retrieved from http://ieeexplore.ieee.org/stamp/stamp.jsp?tp=\&arnumber=4281008.

27. Vasilyeva, E., Pechenizkiy, M., \& Puuronen, S. (2005). Knowledge Management Challenges in Web-based Adaptive E-learning Systems. Retrieved from http://citeseerx.ist.psu.edu/viewdoc/download?doi=10.1.1.91.4296\&rep=rep1\&typ $\underline{\mathrm{e}=\text { pdf. }}$.

28. Wild, R.H., Griggs, K.A., \& Downing, T. (2002). A framework for e-learning as a tool for knowledge management. Industrial management \& Data Systems, 102(7), 371-380.

29. Wu, Y-L., \& Pang, J-J. (2008). Research on the Overall Framework of Knowledge Management. Retrieved from http://ieeexplore.ieee.org/stamp/stamp.jsp?tp=\&arnumber=4680857.

30. Zhan, Q., \& Chang, C. (2008). A Study of E-learning Architecture based on Knowledge Innovation. Computer Science and Software Engineering, 5, 781-784.

31. Zhang, W., \& Kim, M. (2011). Harnessing Explicit Knowledge. Journal of Economics and Behavioral Studies, 2(3), 97-107. 